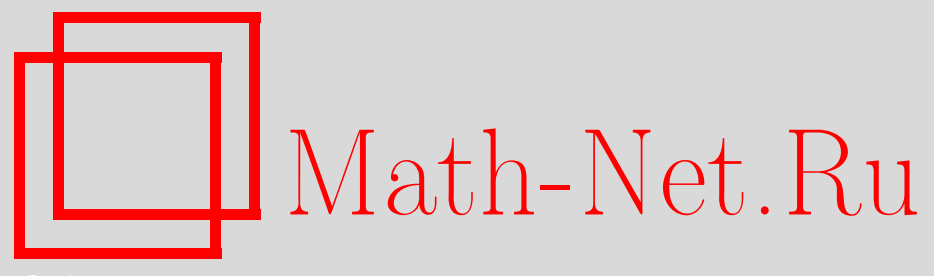

Д. К. Потапов, Аппроксимация однопараметрического семейства задач Дирихле для уравнений эллиптического типа высокого порядка с разрывными нелинейностями в резонансном случае, Матем. заметки, 2011, том 90, выпуск 3, 467-469

DOI: https://doi.org/10.4213/mzm8728

Использование Общероссийского математического портала Math-Net.Ru подразумевает, что вы прочитали и согласны с пользовательским соглашением http://www . mathnet.ru/rus/agreement

Параметры загрузки:

IP : 54.197 .217 .227

26 апреля 2023 г., 14:38:21

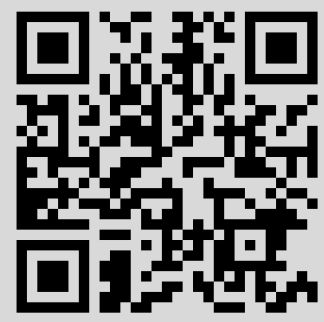




\section{Аппроксимация однопараметрического семейства задач Дирихле для уравнений эллиптического типа высокого порядка с разрывными нелинейностями в резонансном случае}

\section{Д. К. Потапов}

В ряде случаев важен анализ близости решений модели с разрывной нелинейностью и ее аппроксимирующей с непрерывной нелинейностью. Данная проблема была поставлена в работе [1], для коэрцитивных эллиптических краевых задач с разрывными нелинейностями изучалась в работах [2], [3] и в работе [4] для резонансных краевых задач. В работах [5]-[7] рассматривались аппроксимации краевых задач для уравнений эллиптического типа со спектральным параметром и разрывной нелинейностью, а в работе [8] был рассмотрен практический пример - непрерывные аппроксимации задачи об отрывных течениях несжимаемой жидкости Гольдштика. Данная работа является продолжением этих исследований.

В данной работе рассматривается вопрос об аппроксимации задачи Дирихле для уравнения эллиптического типа высокого порядка со спектральным параметром и разрывной нелинейностью в резонансном случае, т.е. случае, когда ядро дифференциального оператора с соответствующим краевым условием ненулевое. Аппроксимирующая задача получается возмущением спектрального параметра и аппроксимацией нелинейности каратеодориевыми функциями. Вариационным методом устанавливается результат о сходимости в равномерной метрике решений аппроксимирующей задачи к решениям исходной задачи. В отличие от работ [2]-[6], [8] в данной работе рассматриваются уравнения высокого порядка, а в отличие от работ [2], [3], [6]-[8] исследуется резонансный случай. Кроме того, в отличие от работ [2]-[4] изучаемые задачи содержат спектральный параметр, в работах [3], [4] рассматривался другой вид аппроксимаций нелинейности.

В ограниченной области $\Omega \subset \mathbb{R}^{n}$ с достаточно гладкой границей $\Gamma$ рассматривается задача Дирихле для уравнения эллиптического типа высокого порядка со спектральным параметром и разрывной нелинейностью вида

$$
\begin{gathered}
\tau u(x)=\lambda g(x, u(x)), \quad x \in \Omega, \\
\partial_{\nu}^{r}(\Gamma) u(x)=0, \quad x \in \Gamma, \quad 0 \leqslant r \leqslant p-1 .
\end{gathered}
$$

Здесь $\tau=\sum_{1 \leqslant|\alpha|=|\beta| \leqslant p}(-1)^{|\beta|} D^{\beta} a_{\alpha \beta}(x) D^{\alpha}$ - равномерно эллиптический формально самосопряженный дифференциальный оператор порядка $2 p$ в дивергентной форме, функции $a_{\alpha \beta}$ вещественнозначны и имеют непрерывные в $\bar{\Omega}$ частные производные до порядка $|\beta|$ включительно; $\lambda$ - положительный параметр; для почти всех $x \in \Omega$ функция $g(x, u)=g_{i}(x, u)$, если $\varphi_{i}(x)<u<\varphi_{i+1}(x), i=1, \ldots, m-1, g(x, u)=g_{0}(x, u)$ при $u<\varphi_{1}(x)$ и $g(x, u)=g_{m}(x, u)$ при $u>\varphi_{m}(x)$, где $g_{i}(x, u)$ - каратеодориевы функции на $\Omega \times \mathbb{R}$, функции $\varphi_{i} \in \mathbb{C}_{2}(\bar{\Omega})$, $i=1, \ldots, m$, удовлетворяют условиям $\varphi_{i}(x)<\varphi_{i+1}(x)$ на $\bar{\Omega}$ для любого $i=1, \ldots, m-1$, и для некоторого $\varepsilon>0$ множества $U_{i}(\varepsilon)=\left\{(x, u) \in \mathbb{R}^{n+1}:\left|u-\varphi_{i}(x)\right|<\varepsilon\right\}, i=1, \ldots, m$, попарно не пересекаются, $g\left(x, \varphi_{i}(x)\right) \in\left[g_{i-1}\left(x, \varphi_{i}(x)\right), g_{i}\left(x, \varphi_{i}(x)\right)\right], i=1, \ldots, m ; \partial_{\nu}^{r}(\Gamma)-$ производные в направлении внутренней нормали $\nu$ к границе $\Gamma$ порядка $r$.

ОПредЕлЕниЕ 1. Силъным решением задачи (1), (2) называется функция $u \in \mathbb{W}_{q}^{2 p}(\Omega)$, удовлетворяющая для почти всех $x \in \Omega$ уравнению (1) и граничным условиям (2).

ОПРЕДЕЛЕниЕ 2. Полуправильным решением задачи (1), (2) называется такое сильное ее решение $u$, значение которого $u(x)$ для почти всех $x \in \Omega$ является точкой непрерывности функции $g(x, \cdot)$.

ОПРеДЕлЕниЕ 3. Прыгающим разрывом функции $f: \mathbb{R} \rightarrow \mathbb{R}$ называется такое $u \in \mathbb{R}$, что $f(u-)<f(u+)$, где $f(u \pm)=\lim _{s \rightarrow u \pm} f(s)$. 
Предположим, что пространство $N(\tau)$ решений задачи

$$
\left\{\begin{array}{l}
\tau u=0 \\
\partial_{\nu}^{r}(\Gamma) u(x)=0
\end{array}\right.
$$

одномерно, $\psi(x)$ - базисная функция этого подпространства. Так как пространство $N(\tau)$ решений задачи (3) ненулевое, имеет место резонансный случай. Пусть для базисной функции $\psi$ пространства $N(\tau)$ верны условия Ландесмана-Лазера

$$
\int_{\psi<0} g_{+}(x) \psi(x) d x+\int_{\psi>0} g_{-}(x) \psi(x) d x>0>\int_{\psi>0} g_{+}(x) \psi(x) d x+\int_{\psi<0} g_{-}(x) \psi(x) d x,
$$

где $g_{ \pm}(x)=\lim _{u \rightarrow \pm \infty} g(x, u)$. Известно [9], что условия Ландесмана-Лазера влекут равенство

$$
\lim _{u \in N(\tau),\|u\| \rightarrow+\infty} \int_{\Omega} d x \int_{0}^{u(x)} g(x, s) d s=-\infty .
$$

Если дополнительно потребовать, что для любого $u \in \mathbb{H}_{\circ}^{p}(\Omega)$ справедливо неравенство

$$
\sum_{1 \leqslant|\alpha|=|\beta| \leqslant p} \int_{\Omega} a_{\alpha \beta}(x) D^{\alpha} u(x) D^{\beta} u(x) d x \geqslant 0
$$

для почти всех $x \in \Omega$ выполнены соотношения $g(x, 0)=0$ и $|g(x, u)| \leqslant a(x)$ для всех $u \in \mathbb{R}$, где $a \in \mathbb{L}_{q}(\Omega), q>\max \{2 n /(n+2 p), n /(2 p)\}$, фиксирована; найдется $u_{0} \in \mathbb{H}_{\circ}^{p}(\Omega)$, для которого имеет место неравенство $\int_{\Omega} d x \int_{0}^{u_{0}(x)} g(x, s) d s>0$, то из теоремы 1 из работы [10] следует существование

$$
\begin{gathered}
0<\lambda_{0} \leqslant \inf _{u_{0} \in U} \frac{\frac{1}{2} \sum_{1 \leqslant|\alpha|=|\beta| \leqslant p} \int_{\Omega} a_{\alpha \beta}(x) D^{\alpha} u_{0}(x) D^{\beta} u_{0}(x) d x}{\int_{\Omega} d x \int_{0}^{u_{0}(x)} g(x, s) d s}, \\
U=\left\{u_{0} \in \mathbb{H}_{\circ}^{p}(\Omega): \int_{\Omega} d x \int_{0}^{u_{0}(x)} g(x, s) d s>0\right\}
\end{gathered}
$$

такого, что для любого $\lambda>\lambda_{0}$ выполняется неравенство

$$
\begin{aligned}
& \inf _{v \in \mathbb{H}_{o}^{p}(\Omega)} J^{\lambda}(v) \\
& \quad=\inf _{v \in \mathbb{H}_{o}^{p}(\Omega)}\left(\frac{1}{2} \sum_{1 \leqslant|\alpha|=|\beta| \leqslant p} \int_{\Omega} a_{\alpha \beta}(x) D^{\alpha} u(x) D^{\beta} u(x) d x-\lambda \int_{\Omega} d x \int_{0}^{u(x)} g(x, s) d s\right)<0,
\end{aligned}
$$

и найдется $u_{\lambda} \in \mathbb{H}_{\circ}^{p}(\Omega)$, для которого

$$
J^{\lambda}\left(u_{\lambda}\right)=\inf _{v \in \mathbb{H}_{O}^{p}(\Omega)} J^{\lambda}(v),
$$

и так как для почти всех $x \in \Omega$ функция $g(x, \cdot)$ имеет только прыгающие разрывы (по построению $\left.g\left(x, \varphi_{i}(x)-\right)=g_{i-1}\left(x, \varphi_{i}(x)\right)<g_{i}\left(x, \varphi_{i}(x)\right)=g\left(x, \varphi_{i}(x)+\right)\right)$, любое $u_{\lambda}$, удовлетворяющее (4), является ненулевым полуправильным решением задачи (1), (2).

Зафиксируем $\lambda>\lambda_{0}$ и пусть числовая последовательность $\left(\lambda_{k}\right), \lambda_{k}>0$, сходится к $\lambda$. Рассмотрим аппроксимирующую задачу

$$
\begin{aligned}
\tau u(x) & =\lambda_{k} g^{k}(x, u(x)), \quad x \in \Omega, \\
\partial_{\nu}^{r}(\Gamma) u(x) & =0, \quad x \in \Gamma, \quad 0 \leqslant r \leqslant p-1,
\end{aligned}
$$


где $g^{k}(x, u)$ - каратеодориева функция на $\Omega \times \mathbb{R}$, равная $g(x, u)$ вне $\bigcup_{i=1}^{m} U_{i}(1 / k), k \in \mathbb{N}$ : $k>1 / \varepsilon$. С каждой задачей (5), (6) свяжем функционал

$$
J_{k}(u)=\frac{1}{2} \sum_{1 \leqslant|\alpha|=|\beta| \leqslant p} \int_{\Omega} a_{\alpha \beta}(x) D^{\alpha} u(x) D^{\beta} u(x) d x-\lambda_{k} \int_{\Omega} d x \int_{0}^{u(x)} g^{k}(x, s) d s
$$

на пространстве $\mathbb{H}_{\circ}^{p}(\Omega)$. Предположим, что для любого $k \in \mathbb{N}$ выполнено неравенство $\left|g^{k}(x, u)\right| \leqslant a(x)$ для всех $u \in \mathbb{R}, a \in \mathbb{L}_{q}(\Omega), q>\max \{2 n /(n+2 p), n /(2 p)\}$. Тогда для любого $k \in \mathbb{N}$ существуют $u_{k} \in \mathbb{H}_{\circ}^{p}(\Omega)$ такие, что $J_{k}\left(u_{k}\right)=\inf _{v \in \mathbb{H}_{\circ}^{p}(\Omega)} J_{k}(v)$, и любое такое $u_{k}$ принадлежит пространству $\mathbb{W}_{q}^{2 p}(\Omega)$ и является сильным решением задачи $(5),(6)$.

Основным результатом данной работы является теорема о близости решений аппроксимирующей задачи (5), (6) к решениям исходной задачи (1), (2).

Теорема 1. Пусть для $\tau, \lambda, g, \lambda_{k}, g^{k}$ выполнены перечисленные выше предположения. Тогда последовательность $\left(u_{k}\right)$ решений аппроксимирующих задач $(5),(6)$, построенная выще, является минимизирующей последовательностью для функционала $J^{\lambda}$ на $\mathbb{H}_{\circ}^{p}(\Omega)$ и содержит подпоследовательность $\left(u_{k_{l}}\right)$, сходящуюся в $\mathbb{C}(\bar{\Omega}) \kappa$ полуправильному решению $\widetilde{u}_{0}$ предельной задачи (1), (2), для которого $J^{\lambda}\left(\widetilde{u}_{0}\right)=\inf _{v \in \mathbb{H}_{o}^{p}(\Omega)} J^{\lambda}(v)$. Если $\widetilde{u}_{0} \in \mathbb{H}_{\circ}^{p}(\Omega)$, удовлетворяющая последнему равенству, единственная, то $u_{k} \rightarrow \widetilde{u}_{0}$ в равномерной метрике $\mathbb{C}(\bar{\Omega})$.

Сформулированная теорема 1 доказывается вариационным методом аналогично теореме 2.1 из работы [5] для резонансных краевых задач эллиптического типа второго порядка со спектральным параметром и разрывной нелинейностью и обобщает результаты работы [5] на уравнения высокого порядка.

\section{СПИСОК ЦИТИРОВАННОЙ ЛИТЕРАТУРЫ}

[1] М. А. Красносельский, А. В. Покровский, Докл. АН СССР, 248:5 (1979), 1056-1059. [2] В. Н. Павленко, Р. С. Искаков, Укр. матем. журн., 51:2 (1999), 224-233. [3] М. Г. Лепчинский, В.Н. Павленко, Алгебра и анализ, 17:3 (2005), 124-138. [4] М. Г. Лепчинский, В. Н. Павленко, Сиб. матем. журн., 46:1 (2005), 139-148. [5] В. Н. Павленко, Д. К. Потапов, Изв. вузов. Матем., 2005, № 4, 49-55. [6] Д. К. Потапов, Изв. РАЕН. Сер. МММИУ, 9:1-2 (2005), 159-165. [7] Д. К. Потапов, Дифферени. уравнения, 43:7 (2007), 1002-1003. [8] Д. К. Потапов, Матем. заметки, 87:2 (2010), 262-266. [9] В. Н. Павленко, В. В. Винокур, Изв. вузов. Матем., 2001, № 5, 43-58. [10] Д. К. Потапов, Дифферени. уравнения, 46:1 (2010), 150-152.

\section{Д. К. Потапов}

Поступило

Санкт-Петербургский государственный университет

01.03 .2010

E-mail: potapov@apmath.spbu.ru 